\title{
Gateway Zone Multi-path Routing in Wireless Mesh Networks
}

\author{
Chun-Wei Chen \\ Dept. of CSIE \\ Natl. Central Univ. \\ Chung-Li, Taiwan, R.O.C. \\ Email: weiwei@wmlab.csie.ncu.edu.tw
}

\author{
Eric Hsiao-Kuang Wu \\ Dept. of CSIE \\ Natl. Central Univ. \\ Chung-Li, Taiwan, R.O.C. \\ Email: hsiao@csie.ncu.edu.tw
}

\begin{abstract}
Internet and multimedia services are widely-provided in All-IP based networks. There has been growing demand for mobile users to get those services. The Wireless Mesh Network (WMN) extends communication range and provides Internet connectivity to mobile users with low upfront investment. In WMN, the traffic usually passes through the gateway and makes it become the common destination of the traffic. Conventional multipath routing protocols in the ad hoc networks do not allow intermediate nodes to reply the route request messages, so the flooding messages make the area near the gateway become congested and degrade the performance. In this paper, we proposed Gateway Zone Multi-path Routing (GZMR) protocol. GZMR uses nodes around the gateway to form a Gateway Cooperative Zone and ceases the routing control messages flooding from outside the zone. Border nodes in the zone can help replying gateway information to reduce route discovery delay. Our simulation results show that by lowering routing overheads and utilizing multiple paths from source to gateway, GZMR can shorten average end-to-end delay and improve the packet delivery in WMNs.
\end{abstract}

\section{Introduction}

In recent years, the rapid growing of the Internet services and on-demand multimedia services in the All-IP networks increase mobile users' demand for acquiring Internet services. The integration between wireless networks and the Internet becomes an important issue. Gateways may be put into wireless ad hoc networks for mobile users connecting to the Internet or other networks. This kind of wireless network is called Wireless Mesh Network (WMN).

General WMNs consist of mesh routers and mesh clients, where mesh routers have minimal mobility and form the backbone of WMNs[1-2]. Mesh clients can be either stationary or mobile devices, and can form a client mesh network among themselves and with mesh routers. WMNs can be classified into three groups: Infrastructure/Backbone WMNs, Client WMNs and Hybrid WMNs. Hybrid WMNs is the most applicable because mesh clients can not only directly communicate with other mesh clients, but also access the Internet service through mesh routers. Many studies [16-17] in the WMNs focus on Infrastructure/ Backbone WMNs. In this paper, we focus on Hybrid WMNs, especially on mesh clients (e.g. Mobile Ad Hoc Network (MANET)) accessing Internet service through gateway supported mesh routers.

MANETs are autonomous, infrastructure-less networks that support multi-hop communications through IP routing and mostly used for military purpose. MANETs are characterized by dynamic topology due to mobility, limited channel bandwidth and limited battery power of nodes. In MANETs, the information is exchanged mostly between users such as playing inactive games. However, in WMN, it allows users to access the information in the Internet through gateways. When MANET has connectivity to the Internet, major user behaviors are to access Internet services (e.g. browsing webpage, checking e-mails or downloading files) and multimedia services. We could refer to the lately studies [3, 4], which show that while clients are accessing Internet service through Access Points (APs). And most of the trafficcs are HTTP traffics.

In order to provide certain Quality of Service (QoS) for mobile users to access the Internet and multimedia service, there are singlepath and multipath routing protocols proposed and discussed. Singlepath routing protocols $[5,6]$ are easy to implement but it may suffer from frequently route discovering and cause significant latency and routing overheads when the mobility increases. Multipath routing [7-15] aims to build multiple paths to increase fault tolerance and further reduce routing overheads. When taking Internet connectivity into consideration, the traditional multipath routing protocols may encounter routing control messages flooding problem and gateway congested problem. The former is caused because that most of these protocols close the mechanism that intermediate nodes reply to the route request, so route request messages flood to the gateway and areas near the gateway become congested. The latter is resulted form routing control messages flooding and additional control messages processing. The gateway has to spend more time dealing with route request messages when relaying data packets. 
In this paper, we present Gateway Zone Multi-path Routing (GZMR) protocol that nodes around the gateway form a Gateway Cooperative Zone to cease the routing control messages flooding from outside the zone and solve routing control messages flooding problem. And Border Nodes in the zone can help replying gateway information to nodes outside the zone to solve gateway congested problem. The reducing routing overheads and multiple paths from source to the gateway can help reducing average end-to-end delay and improving the packet delivery in wireless mesh networks.

The rest of this paper is organized as follows. In Section 2, we review the related work of conventional multipath routing protocols in MANETs. We propose our GZMR protocol in Section 3. In Section 4, the performance evaluation is presented through ns2 simulation and we concluded this paper in Section 5.

\section{Related work}

Multipath routing is firstly designed for load balancing in the wired networks to achieve the Quality of Service (QoS). In MANET, the communication is prone to broken because of the dynamic topology. Multipath routing benefits this kind of networks not only load balancing, but also fault tolerance. A node may switch to the backup paths when the primary path is broken, which help reduce the route rediscovery and lower the routing overhead, and further reduce the average end-to-end delay and packet loss.

SMR[10] and MSR[11] share lots of similarity to DSR. SMR intends to construct multiple maximally disjoint paths. MSR and SMR are much alike, except that MSR take hop-count of the paths into consideration and has a scheme for the paths using. There is a traffic allocation scheme proposed for distributing packets into multipath according to the weight of a path. Both of them only allow the destination to reply the Route Requests (RREQs), and intermediate nodes are not allowed to send Route Replies (RREPs) back to the source even when they have route information to the destination.

AOMDV[12] is an on-demand multipath routing protocol based on AODV and intends to compute multiple loop-free and link-disjoint paths. The source floods RREQs when it needs routes to delivery packets to the destination. When receiving duplicate RREQs, intermediate nodes record the RREQs information without discarding them right away. For each destination, the node keeps multiple paths and all of them have the same sequence number and it maintains advertised hop count, which is the max hop count of all the paths. Because of the scheme of finding link disjoint paths, AOMDV may encounter the route cutoff problems when establishing reverse paths from the destination back to the source.

AODVM[13] extending form AODV is an on-demand routing protocol for finding multiple node-disjoint paths. Intermediate are not permitted to reply to RREQs. They do not discard duplicated RREQs immediately, but record the information contained in RREQs into the RREQ table instead. The destination replies to RREQs and deletes the sending neighbors from the RREQ table. Like the destination, intermediate nodes also delete the sending neighbors from the RREQ table and then find the shortest path in the routing table to forward it. To ensure all the finding paths are node-disjoint, every node deletes the neighbor from the RREQ table when overhearing it broadcasts the RREP message.

CHAMP[15] aims to reduce packet loss in MANETs by using cooperative packet caching and utilizing multiple shortest paths. CHAMP allows for non-disjoint paths. The source floods RREQs when there is no route to the destination. The destination sends RREPs back to the source when the hop-count received RREQs are not larger than the shortest paths ever created. Packets were then sent to the multiple paths in turn to keep the paths fresh. And the paths with equal lengths help eliminate packet out-of-order problems. Packet caching scheme salvages packets by sending them to the backup paths. If no alternative paths exist, it broadcasts a RERR message with packet information. The upstream nodes who receive the RERR will check their routing table and packet cache to make sure they have alternative paths and cached packets to salvage. Otherwise they re-broadcast the RERR message. The source will re-initiate route discovery if it receives RERR without having any alternative paths.

Global6[18] describes how to provide Internet connectivity to MANET. In [19], the author implements necessary parts of Global6 in NS2. The study explains how MN and Gateway should operate, and propose how to apply a method for discovering gateways. Extend the AODV route discovery messaging, so it can be used for discovering not only mobile nodes but also gateways. Routing messages RREQ_I and RREP_I, whose I-flag represents Internet-Global Address Resolution Flag, are used to get gateways' information. Three kinds of gateway discovery mechanisms are described in this project and the gateway advertisement (GWADV) message, a new AODV message, has been introduced.

In WMNs, the traffic's direction is from WMNs to the wired nodes to access the Internet services. Thus, gateways become common destinations. In most of multipath routing protocols $[10,11,13,15]$, intermediate nodes are not allowed to reply to the RREQs, so the RREQs maybe flooded to the whole network until they get to the destination. The gateway is the common destination and the dramatic increasing of routing overheads will flood around it. Besides, the gateway will increase additional delay in forwarding packets due to busy in handling routing control packets. Since the bandwidth in wireless ad hoc networks is limited, how to reduce routing overhead has to be considered when designing a routing protocol.

\section{Gateway zone multi-path routing}

\subsection{Overview}


Our objective in this section is to design an efficient multipath routing mechanism, Gateway Zone Multi-path Routing (GZMR), to help reducing routing overheads around the gateway and improving packet delivery in the wireless mesh network.

\subsection{System model}

\subsubsection{System architecture}

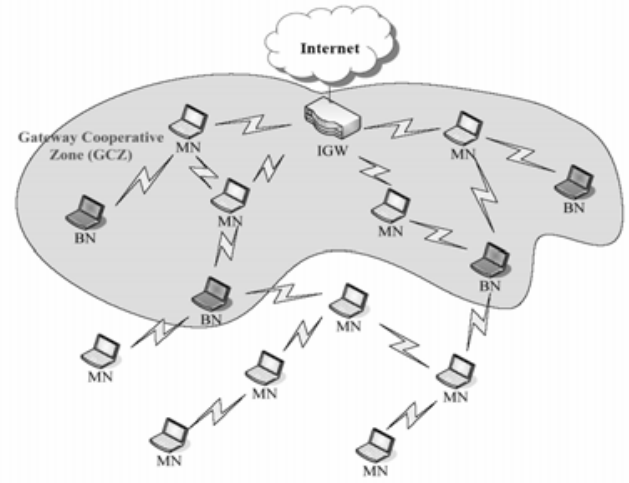

Figure 1. System Architecture of GZMR.

First of all, we introduce the system architecture of our scheme in Figure 1. It is a hybrid wireless mesh network that mesh clients connect to a mesh router with gateways support (or Internet Gateway) to access Internet service. We define a Gateway Cooperative Zone (GCZ), which is formed by two or three hops neighbors of the Internet Gateway (IGW). Border Nodes (BNs) are in the rim of GCZ. Mobile Nodes (MNs) and BNs can communicate to Fixed Nodes (FNs) in the Internet. BNs act as guard nodes, which restrain Route Request (RREQ) messages flooding into GCZ.

Figure 2: Protocol stacks of mobile nodes, Internet gateways and fixed nodes. The network layer is coordinated by GZMR (Figure 3), composed by zone formation, gateway/route discovery, neighbor history record (NHR) and data forwarding.

\subsubsection{Message formats}

According to [19-20], we use RREQ_I and RREP_I, which are Route Request (RREQ) message with Internet-Global Address Resolution Flag (I-flag) and Route Reply (RREP) with I-flag in brief to support gateway discovery operations. The purpose of RREQ_I message is to reactively discovery a gateway.

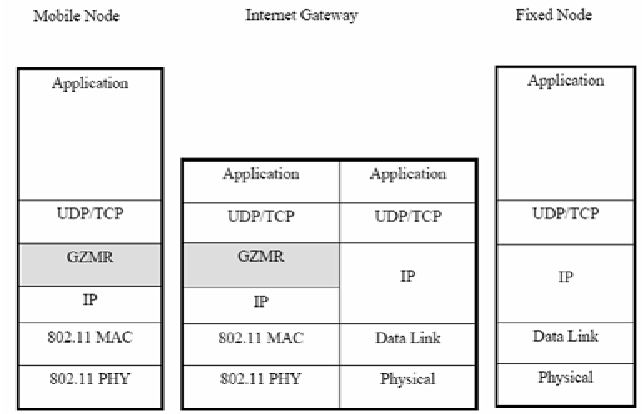

Figure 2. Protocol stacks of mobile nodes, Internet gateways and fixed nodes.

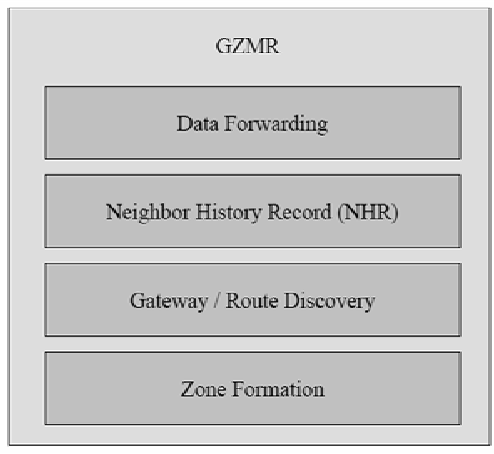

Figure 3. Protocol stacks of GZMR.

There are various types of nodes defined in GZMR. They are GW_NODE, GCZ_NODE, BN_NODE and USUAL_NODE. We further design routing messages, such as Route Request with B-flag (RREQ_B), Route Reply with B-flag (RREP_B) and Route Reply with BI-flag (RREP_BI), to process route discovery from different types of nodes. The purpose of RREQ_B is for nodes in the GCZ to request for a route to unknown destinations, and RREP_B is for GW_NODE and GCZ_NODE to build reverse paths to the mobile sources. RREP_BI is a combined message of RREP_B and RREP_I sent by BN_NODE when receiving RREQ from USUAL_NODE and having no route information to destination.

Figure 4 shows the message format of Gateway Advertisement (GWADV) broadcasted by IGW, and the Neighbor Cache in the GWADV is used for BNs (or BN_NODEs) to keep tracks of GCZ_NODEs.

\begin{tabular}{|c|c|c|c|}
\hline & & & \\
\hline Type & Reserved & Prefix Sz & Hop Count \\
\hline \multicolumn{4}{|c|}{ RREQ ID } \\
\hline \multicolumn{4}{|c|}{ Destination IP Address } \\
\hline \multicolumn{4}{|c|}{ Destination Sequence Number } \\
\hline \multicolumn{4}{|c|}{ Source IP Address } \\
\hline \multicolumn{4}{|c|}{ LIFETIME } \\
\hline & Neigh & & \\
\hline
\end{tabular}

Figure 4. Gateway Advertisement (GWADV) Message Format.

\begin{tabular}{|c|c|c|}
\hline MN_src & \multicolumn{2}{|c|}{ History Record } \\
\hline \multirow{4}{*}{} & nexthop1 & latest_use_time 1 \\
\cline { 2 - 3 } & nexthop2 & latest_use_time \\
\cline { 2 - 3 } & & \\
& & \\
\hline
\end{tabular}

Figure 5. Neighbor History Record in GZMR.

\subsubsection{Routing table and neighbor history record}

The routing table used in GZMR is similar to AOMDV. Each entry in the routing table includes the destination's address, sequence number, multiple paths to the destination and the max hop-count of all the paths. We create a timer to periodically purge expired paths to avoid using stale paths.

Neighbor History Record (NHR) is designed for GCZ_NODE to keep tracks of the directions where the data packets came from. The purpose of using NHR is to 
quickly recover downstream connection and avoid RREQ_B flooding. GCZ_NODE who has the record in its NHR will unicast RREQ_B to find paths to the mobile nodes. See Figure 5.

\subsection{Gateway Discovery}

In GZMR, We apply hybrid gateway discovery mechanism that IGW periodically broadcasts the Gateway Advertisement (GWADV) message. Nodes inside GCZ (GCZ_NODEs) can obtain IGW information and establish multiple paths to default IGW from receiving GWADVs. BNs will record the Neighbor Cache in each received GWADV into its routing table.

\subsection{Route discovery}

The mechanisms of discovering routing paths are different from the nodes in GCZ and those outside GCZ. The nodes in GCZ will be fully-meshed connected. The others outside GCZ will have routing paths which are link-disjointed.

\subsubsection{Mobile nodes communicate to fixed nodes}

a) Mobile nodes outside GCZ: In order to make an on-demand connection with Fixed Nodes (FN) in the wired network, the mobile node outside the GCZ (USUAL_NODE) will broadcast the Route Request (RREQ) message when it doesn't know where the destination is. When the RREQs are flooded to BNs (BN_NODEs), each BN firstly checks its routing table and broadcasts RREP_BI if it has no information about the destination. When GCZ_NODE receives RREP_BI, it establishes multiple fully meshed paths to source, converts RREP_BI to RREP_B and rebroadcasts it to IGW. The USUAL_NODE who receives a RREP_BI converts RREP_BI to RREP_I and establishes forward paths to IGW. The way of broadcasting RREP_BI is to build the reverse paths to the sources for the IGW in advance, because we know most of the connections related to accessing the Internet services are interactive.

After receiving RREP_I, the source establishes multiple link-disjoint forward paths to BNs. After the timeout event of a RREQ occurs but no RREPs are received, the source will then determine that the destination is a FN in the wired network and forward data packets to IGW. Figure 6 shows the procedure of USUAL_NODE communicating to FN.

b) Mobile nodes inside GCZ: We use B-flag to distinguish the route request sent by nodes inside GCZ from that sent by nodes outside GCZ. When making an on-demand connection with FN, the node in the GCZ (e.g. BN_NODE, GW_NODE and GCZ_NODE) will send the Route Request with B-flag (RREQ_B) messages when it doesn't know where the destination is. While receiving the RREQ_B, GW_NODE can reply to it if it has route to the requested destination; GCZ_NODE and BN_NODE can also reply to it if they have routes and the requested destination is not a FN. If the source gets no reply from the requested destination after the timeout event of the RREQ_B occurs, it then determines

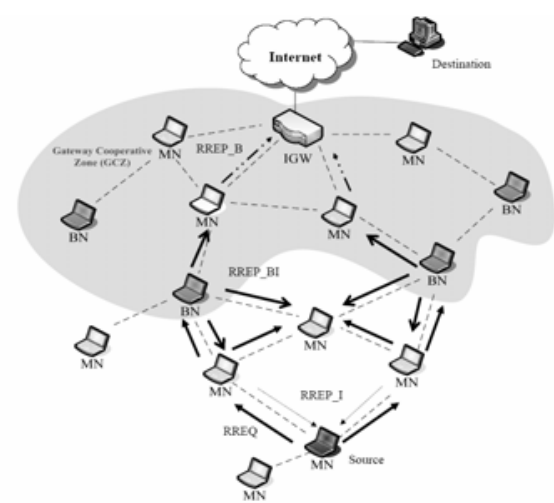

Figure 6. Route Discovery for Mobile nodes outside GCZ.

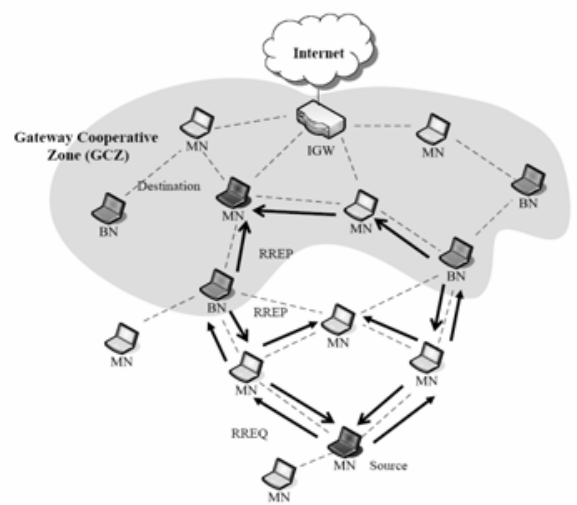

Figure 7. Route Discovery for Mobile nodes to Mobile nodes.

that the destination is a FN in the wired network.

\subsubsection{Mobile nodes communicate to mobile nodes}

We take the situation when USUAL_NODE communicates to GCZ_NODE into consideration. The entries in the BNs' routing table established when receiving GWADV are helpful. BNs can quickly reply to USUAL_NODE and build paths to GCZ_NODE. See Figure 7. However, when GCZ_NODE communicates to USUAL_NODE, the route discovery procedure is similar to the first time GCZ_NODE communicate to FN by broadcasting RREQ_B. When USUAL_NODE destination receives the RREQ_Bs, it sends RREPs back to the GCZ_NODE.

\subsubsection{Down-stream route discovery}

When the traffic is from $\mathrm{FN}$ to $\mathrm{MN}$, downloading data from the Internet for example, the IGW becomes a bottle-neck. The paths broke easily most of the time because of the collision rather than node mobility. Here we design a NHR Module to handle this situation.

a) Original GZMR: IGW Broadcast RREQ B in GCZ: The original way is the same as AODV and AOMDV. In order to find a route, IGW broadcasts RREQ_B in GCZ. And any node which has routes to the destination can reply the RREQ $\_$, or the RREQ_B will be re-broadcasted to the requested destination. See Figure 8. 


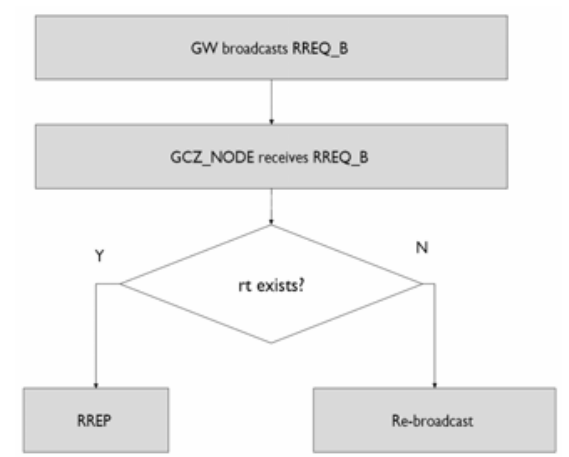

Figure 8. Original GZMR.

b) GZMR with NHR Module: Use Node History Record (NHR): The Neighbor History Record (NHR) is used to help reducing the broadcasting RREQ_B messages in GCZ. GCZ_NODEs keep tracks of the directions where the data packets are coming from. According to NHR, downstream connections can be quickly recovered by GCZ_NODE unicasting RREQ_B to find routes to MN source. If there are no other replies back to IGW, IGW will set the through-flag to 1 and broadcast the RREQ_B again. The rest of steps are the same as Original GZMR. See Figure 9.

\subsection{Data forwarding}

In GZMR, a node always chooses the smallest hop-count path when forwarding data packets. If there are same hop-count paths, the node will choose the fresher one. This a little bit different form AOMDV, which uses paths according to the path built sequence. When a node detects the using path is broken, it then switches to another path to salvage packets.

\subsection{Route maintenance}

When forwarding a data packet, the node uses data packet acknowledgment to judge the status of the link. The link is "DOWN" when there is no data packet acknowledgments back. If there is no other paths exist, the node will re-discovery the route if it is the source of the traffic, and if it is a intermediate node, it will simply drop the data packet and broadcast the Route Error (RERR) message to its neighbors.

\section{Performance evaluation}

\subsection{Simulation environment}

The simulation environment was created in the famous network simulator NS-2 to develop and analyze the proposed protocol GZMR. There are total 60 mobile nodes, one Gateway and 10 fixed nodes in a $1500 \mathrm{~m} \mathrm{x}$ $750 \mathrm{~m}$ topology with Gateway placed at $(1450,380)$. We use traffic generator to develop the simulated TCP and CBR traffic sources, and use Random way-point model as the mobility model. The channel capacity is $11 \mathrm{Mbps}$ and we use the IEEE 802.11 Distributed Coordination Function (DCF) as the medium access control protocol. We use AOMDV+ as samples for comparison. We

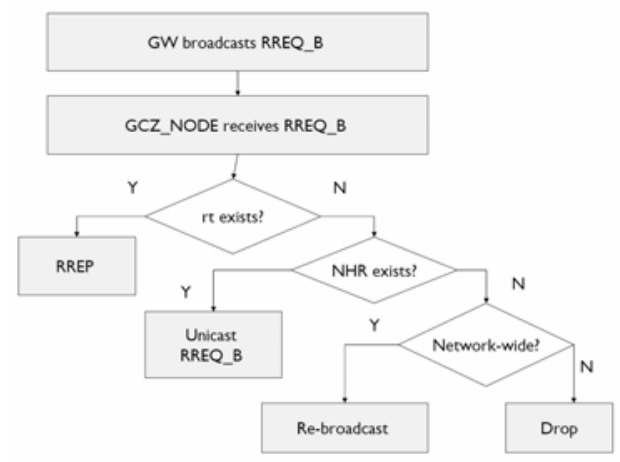

Figure 9. GZMR with NHR

modified AOMDV[12] to AOMDV+ with the ability to have gateway support according to AODV+[19], which is developed by Ali Hamidian to extend AODV to have gateway support. In the following simulations we evaluate CBR and TCP traffics by varying the node mobility and the number of connections.

\subsection{Performance metrics}

We use the following metrics to evaluate the protocols in varying scenarios:

Packet Delivery Ratio: The ratio of the total number of data packets received by the destination to the total number of packets sent by the source.

Average End-to-end Delay: The average end-to-end delay is the average delay between the time at which the data packet was originated at the source and the time it was received by the destination. Delay includes route discovery latency, propagation delay, queuing delay and retransmissions. Note that data packets that get lost are not considered.

Normalized Routing Load: The normalized routing load is defined as the total number of routing control packets divide by the total number of data packets received by the destination. It presents the efficiency of the protocol.

\subsection{Simulation results}

\subsubsection{Performance of varying GCZ size in GZMR}

In order to analyze the impact of GCZ size, we first set up a simulation environment and vary the size of GCZ to see the effect on the packet delivery ratio, normalized routing load, average end-to-end delay, and route discovery duration. The traffics we generate in this scenario are CBR with the direction from mobile nodes to the fixed nodes through gateway. We vary the number of CBR connections with the maximum speed $5 \mathrm{~m} / \mathrm{s}$. The pause time is $100 \mathrm{~s}$, and the total simulation time is $300 \mathrm{~s}$. We vary the GCZ size from 1 to 3 hop counts in this experiment due to the average hop counts of each connection are 6 or 7 .

The increasing number of connections causes much more link broken and also produces more routing overheads. As we can see in Figure 10, when the GCZ size is larger, the packet delivery ratio and normalized routing load (Figure 11) are better because of less 
routing messages will flood around the gateway. The average end-to-end delay (Figure 12) is longer when GCZ size is smaller, because that BNs and the gateway are too close to each other and may encounter more interference.

According to this experiment, the performance is better when GCZ size is larger, we choose hop count $=3$ in the rest of our experiments.

\subsubsection{Performance of CBR traffic from MN to FN}

In this experiment, we vary the number of CBR connections with the maximum speed $5 \mathrm{~m} / \mathrm{s}$. The Pause time is $50 \mathrm{~s}$, and the total simulation time is 300s. The increasing of the number of connections causes more routing overheads when the links break. As we can see in Figure 13, the packet delivery ratio for AOMDV+ decreases as compared to GZMR for higher number of connections. The ratio drops further in AOMDV+. But GZMR still has better performance than AOMDV+. This is because the aggressive routing overheads of AOMDV+ increase the routing load in gateway. As can be seen from Figure 14 the normalized routing load of AOMDV+ is higher than GZMR. Average end-to-end delay value for GZMR is better than AOMDV+ under all conditions in Figure 15. The difference becomes significant at larger number of connections because of the decrease in the routing overhead.

\subsubsection{Performance of TCP traffic from MN to FN}

We simulate the TCP traffics with the direction from mobile nodes to the fixed nodes through the gateway. The purpose of this scenario is to simulate the situation of mobile users uploading information to the wired network, for example, users can upload their diaries and photos to the web blogs. The data packet size is set to be 1460 bytes.

In this experiment, we vary the number of TCP connections with the max speed $5 \mathrm{~m} / \mathrm{s}$. The Pause time is $50 \mathrm{~s}$, and the total simulation time is 300s. The increasing traffic load leads to larger control overheads, then causes longer average end-to-end delay due to the packets queuing delay when source performs route discovery frequently. In Figure 16 and Figure 17, GZMR has better packet delivery ratio and less normalized routing load than AOMDV+. Because less congestion and few links broken around GCZ are caused, thus reduce packet transmission delay (Figure 18).

\subsubsection{Performance of Bi-directional TCP Traffic}

We simulate the TCP traffic with both directions from mobile nodes and the fixed nodes through the gateway and vice versa. The purpose of this scenario is to simulate the situation of mobile users requesting for the Internet services. The data packet size is set to be 500 bytes from $\mathrm{MN}$ to $\mathrm{FN}$ and 1460 bytes from FN back to MN.

We generate 10 pairs of TCP connections with the $\max$ speed $10 \mathrm{~m} / \mathrm{s}$. The Pause time varies from 0s to 300 s, and the total simulation time is 300 s. As can be

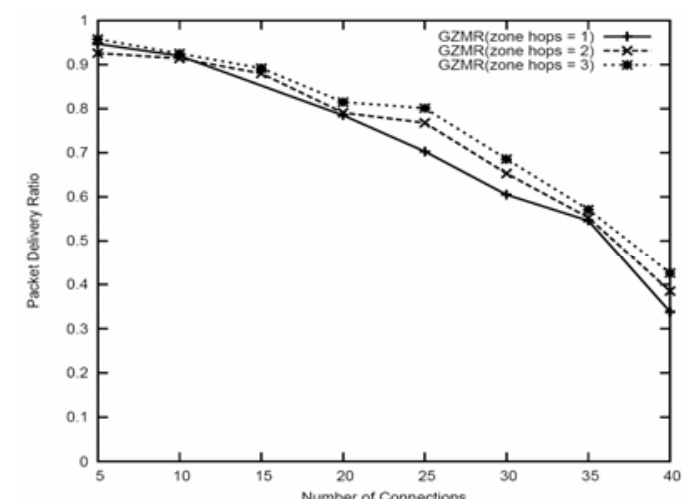

Figure 10. Packet Delivery Ratio vs. Connection

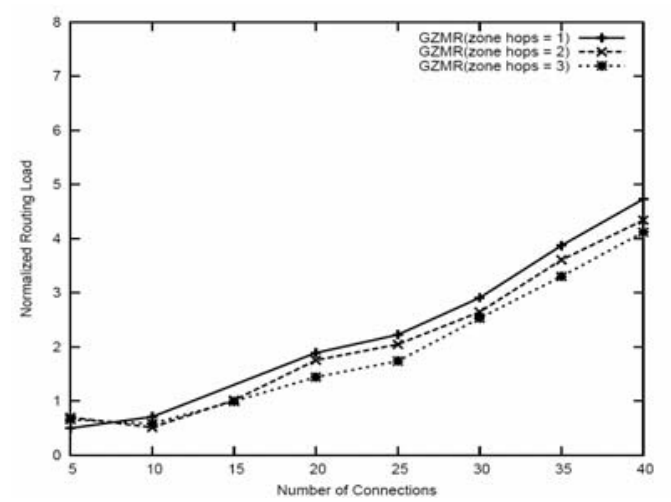

Figure 11. Normalized Routing Load vs. Connections

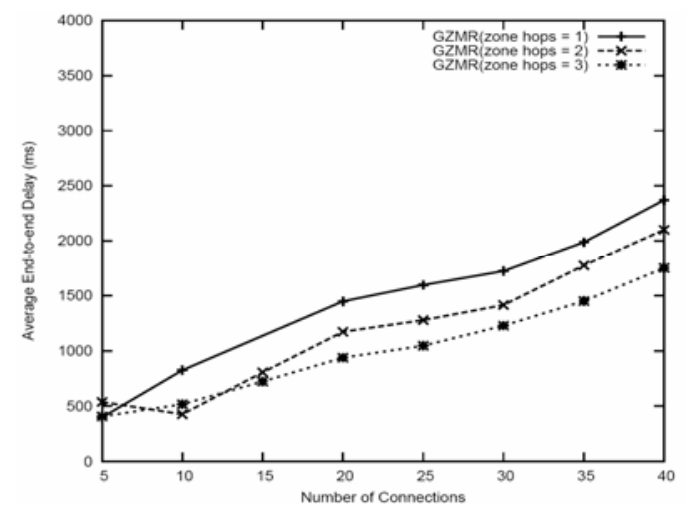

Figure 12. Average End-to-End Delay vs. Connections

seen from Figure 19, GZMR can still achieve higher sent packets than AOMDV+. Since the traffic is bidirectional, there are data packets and ACKs sent to and from the gateway much frequently. So the large traffic load in the gateway may cause the down-stream links break easily, and thus causes much more congestion around the gateway. In the Figure 20, all of the packet delivery ratios of these two protocols are relatively low, but GZMR outperforms the others a little bit. In Figure 21 and Figure 22, we can see that GZMR earns shorter delay and less normalized routing load and aids in reduction the traffic load around gateway to achieve higher performance. AOMDV+, on the other hand, needs to wait route discovery responses from the gateway and has longer delay. 


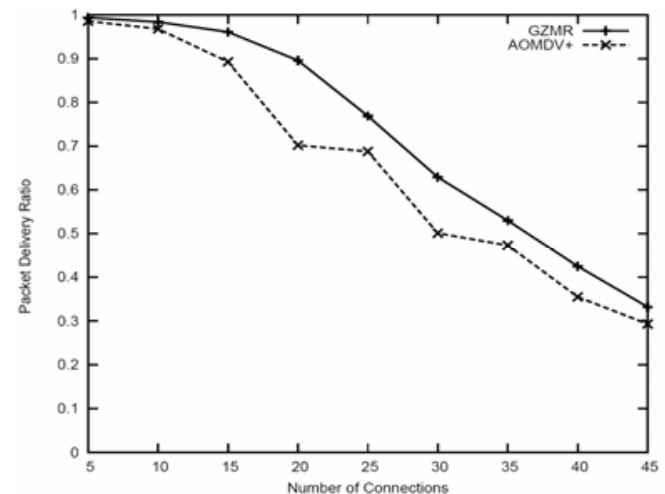

Figure 13. Packet Delivery Ratio vs. Connections (CBR Traffic)

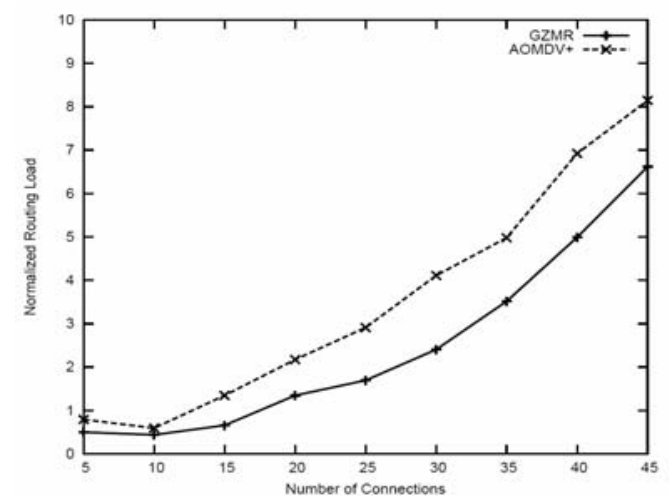

Figure 14. Normalized Routing Load vs. Connections (CBR Traffic)

\section{Conclusions and future work}

We proposed the Gateway Zone Multi-path Routing (GZMR) in Wireless Mesh Networks to reduce routing overheads around IGW, which is the destination of most of the traffics. Our scheme uses Border Nodes to effectively cease RREQs being flooded from outside Gateway Cooperative Zone (GCZ) and act as relay nodes to reply gateway information to mobile nodes outside GCZ. As can be see from the experiment results, GZMR provides lower latency and higher performance than AOMDV when accessing the Internet services in Wireless Mesh Network.

As a future work, we intend to use more metrics (e.g. Node stability, residual power, etc.) to determine the GCZ and BN. To make our proposed protocol more adaptive to real situations, we plan to simulate various kinds of applications (e.g. E-mail, Video on-demand, etc.) to evaluate the performance of our protocol. Finally, the further research to apply our protocol to different networks is needed. We intend to implement it not only in 802.11 but also in 802.16 WiMAX.

\section{References}

[1] W. X. Akyildiz, I.F. and W. Wang, "Wireless mesh networks: A survey”, Computer Networks Journal (Elsevier), March 2005.

[2] P. Whitehead, "Mesh networks; a new architecture for broadband wireless access systems", Proc. of IEEE Conference on Radio and Wireless, pp. 43-46, 10-13 Sept. 2000.

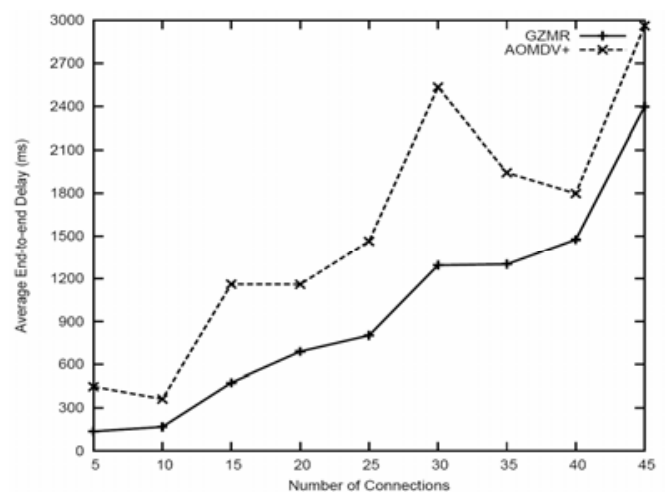

Figure 15. Average End-to-End Delay vs. Connections (CBR Traffic)

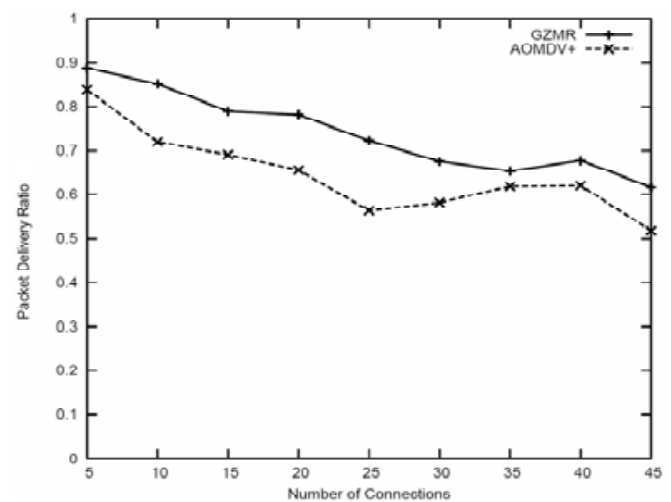

Figure 16. Packet Delivery Ratio vs. Connections (TCP Traffic)

[3] P. B. A. Balachandran, G. M. Voelker and P. V. Rangan, Characterizing user behavior and network performance in a public wireless LANProc. of Int. Conf. Measurement and Modeling Computer Systems (SIGMETRICS), Marina. del Rey, CA, pp. 195-205, Jun. 2002.

[4] J. C. C. Na and T. Rappaport, "Hotspot traffic statistics and throughput models for several applications", Proc. of IEEE GLOBECOM, Vol. 5, pp. 3257-3263, December 2004.

[5] D. Johnson and D. Maltz, ".Dynamic source routing in ad hoc wireless networks”, Mobile Computing, Chapter 5, ed. T. Imielinski and $\mathrm{H}$. Korth, Kluwer Academic Publishers, pp. 153-181, 1996.

[6] E. R. C.E. Perkins, "Ad-hoc on-demand distance vector routing", Proc. of IEEE Workshop on Mobile Computing Systems and Applications (WMCSA), pp.99-100, 1999.

[7] D. G. S. Mueller, "Multipath routing in mobile ad hoc networks: issues and challenges", Performance Tools and Application to Networked Systems, ser. Lecture Notes in Computer Science, M.C. Calzarossa and E. Gelenbe, Eds., vol. 2965, pp. 209-234, Apr. 2004.

[8] S. J. Lee and M. Gerla, "AODV-BR: Backup routing in ad hoc networks", Proc. of IEEE WCNC 2000, Chicago, USA, Vol. 3, pp. 1311-1316, September 2000.

[9] K. Wu and J. Harms, "Performance study of a multipath routing method for wireless mobile ad hoc networks", Proc. of Symposium on Modeling, Analysis and. Simulation on Computer and Telecommunication Systems, pp. 99-107, August 2001.

[10] S. Lee and M. Gerla, "Split multipath routing with maximally disjoint paths in ad hoc networks”, Proc. IEEE International Conference on Communications (ICC)'01 , pp. 3201-3205, June 2001. 


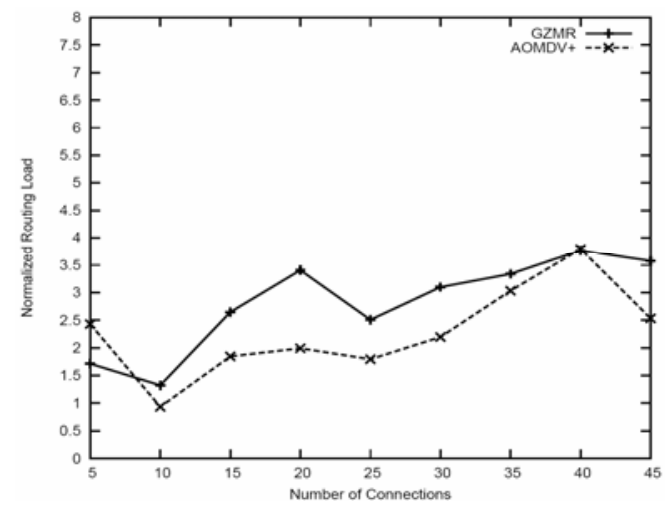

Figure 17. Normalized Routing Load vs. Connections of (TCP Traffic)

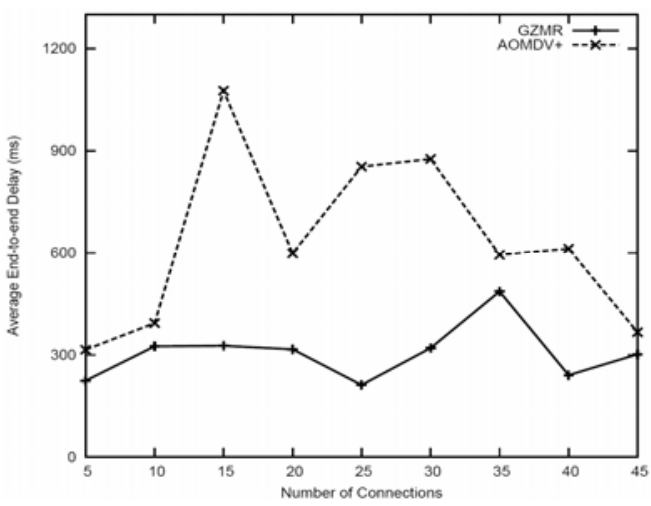

Figure 18. Average End-to-End Delay vs. Connections (TCP Traffic)

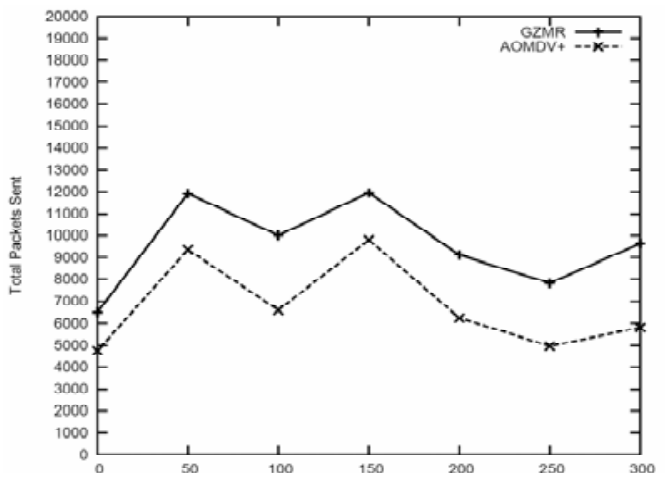

Figure 19. Total Sent Packets vs. Pause Time (Bi-TCP Traffic)

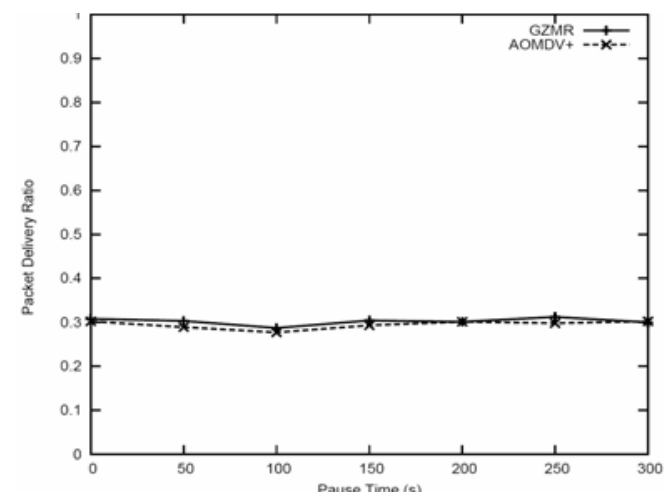

Figure 20. Packet Delivery Ratio vs. Pause Time (Bi-TCP Traffic)

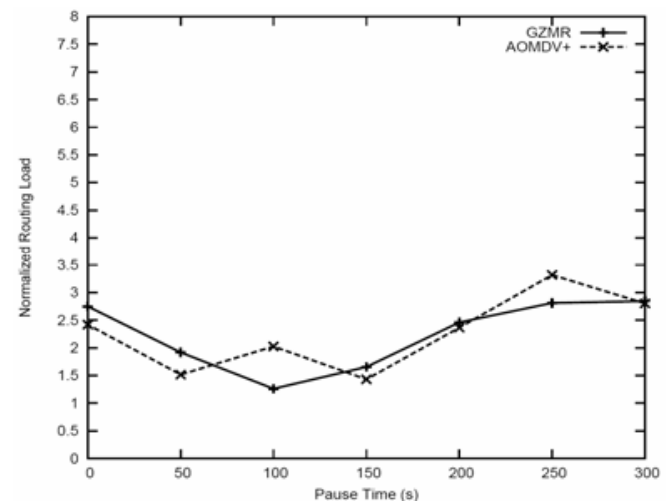

Figure 21. Normalized Routing Load vs. Pause Time (Bi-TCP Traffic)

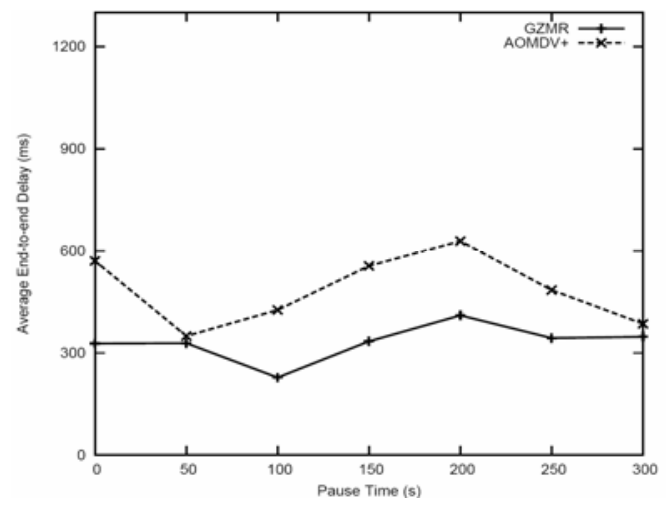

Figure 22. Average End-to-End Delay vs. Pause Time (Bi-TCP Traffic)

[11] L. Wang, Y. Shu, M. Dong, L. Zhang, and O. Yang, "Adaptive multipath source routing in ad hoc networks", Proc. of IEEE Int'l Conf. on Communications, vol.3, pp. 867-871, Jun. 2001.

[12] M. K. Marina and S. R. Das, "On-demand multipath distance vector routing in ad hoc networks", Proc. of IEEE International Conference on Network Protocols (ICNP), pp. 14-23, 2001.

[13] S. K. Z. Ye and S. Tripathi, "A framework for reliable routing in mobile ad hoc networks”, Proc. of IEEE INFOCOM, vol.1, pp. 270-280, Apr. 2003.

[14] Y. Z. Y. Lu, W. Wang and B. Bhargava, "Study of distance vector routing protocols for mobile ad hoc networks", Proc. of IEEE International Conference on Pervasive Computing and Communications, pp. 187, 2003.

[15] S.R., A. Valera, and W. Seah, "Improving protocol robustness in ad hoc networks through cooperative packet caching and shortest multipath routing”, IEEE Transactions on Mobile Computing, vol. 04, no. 5, pp. 443-457, Sept. 2005.

[16] B. Z. R. Draves, J. Padhye, "Comparisons of routing metrics for static multi-hop wireless networks," ACM Annual Conference of the Special Interest Group on Data Communication (SIGCOMM), pp. 133-144, August 2004.

[17] B. Z. R. Draves, J. Padhye, "Routing in multi-radio, multi-hop wireless mesh networks," ACM Annual International Conference on Mobile Computing and Networking (MOBICOM), pp. 114-128, 2004.

[18] R. Wikikawa, J. T. Malinen, C. E. Perkins, A. Nilsson, and A. J. Tuominen, "Global connectivity for ipv6 mobile ad hoc networks”, IETF Internet Draft, draft-wakikawa-manetglobalv6-00.txt, Workingprogress, Nov. 2001.

[19] A. Hamidian, "A study of internet connectivity for mobile ad hoc networks in ns2", Masters thesis. Department of Communication Systems, Lund Institute of Technology, Lund University, Jan. 2003. 